\title{
Constraint Violation Stabilization Using Gradient Feedback in Constrained Dynamics Simulation
}

\author{
Sugjoon Yoon* \\ Korea Institute of Aeronautical Technology, Korean Air, Seoul, Korea \\ and \\ Robert M. Howe $\dagger$ and Donald T. Greenwood $\dagger$ \\ University of Michigan, Ann Arbor, Michigan 48109
}

\begin{abstract}
Conventional holonomic or nonholonomic constraints are defined as geometric constraints in this paper. If the total energy of a dynamic system can be computed from the initial energy plus the time integral of the energy input rate due to external or internal forces, then the total energy can be artificially treated as a constraint. The violation of the total energy constraint due to numerical errors during simulation can be used as information to control these errors. When geometric constraint control is combined with energy constraint control, numerical simulation of a constrained dynamic system becomes more accurate. An energy constraint control based on the gradient feedback of the energy constraint violation leads to a new method to control both geometric and energy constraint violations, so-called constraint violation stabilization using gradient feedback. A new convenient and effective method to implement energy constraint control in numerical simulation is developed based on the geometric interpretation of the relation between constraints in the phase space. Several combinations of energy constraint control with either Baumgarte's constraint violation stabilization method or the new constraint violation stabilization using gradient feedback are also addressed. Finally, a new method for implementing constraint controls is developed by using the Euler method for integrating constraint control terms, even when higher-order integration methods are used for all other integrations.
\end{abstract}

\section{Introduction}

$\mathbf{T}$ HE dynamics of multibody systems, as they arise in robotics, biomechanics, space vehicle dynamics, and other applications, are becoming increasingly important. Traditionally, the task of formulating the equations of motion has been of dominant concern in the literature. ${ }^{1-3}$ The Lagrange multiplier method ${ }^{4}$ for solving constrained dynamic systems has certain advantages over other methods because the selection of generalized coordinates and the analysis of constraint forces are relatively straightforward, even though the evaluation of the constraint forces can involve much computation if the system is complex. In the analysis of constrained holonomic systems, ${ }^{4,5}$ the Lagrange multiplier method yields a system of second-order ordinary differential equations of motion and algebraic constraint equations. For nonholonomic systems with linear constraints in the velocities, the Lagrange multiplier method yields second-order ordinary differential equations of motion and first-order ordinary differential constraint equations. The Lagrange equations of motion, with the accompanying constraint equations for holonomic or nonholonomic systems, cannot, in general, be solved analytically.

Theoretical analyses ${ }^{6-9}$ for general Lagrange equations with algebraic constraint equations show that constraint equations should be differentiated twice, in general, for the system to be solved numerically without iteration. The differentiation of constraint equations was suggested ${ }^{10}$ prior to these analyses and was shown to result in unstable numerical solutions. The original constraint equations are rapidly violated, since the differentiated constraint equations are unstable and numerical errors during computation continuously disturb the system. ${ }^{11}$ This problem was seemingly resolved by a modified form of

Received July 3, 1991; revision received Feb. 15, 1992; accepted for publication March 20, 1992. Copyright (C) 1992 by the American Institute of Aeronautics and Astronautics, Inc. All rights reserved.

*Research Specialist, 118, 2-ka, Namdaemun-ro, Chung-ku, Seoul, Korea.

$\dagger$ Professor, Department of Aerospace Engineering. second-order differential constraint equations. But this solution, the constraint violation stabilization method (CVSM), ${ }^{12}$ did not work well for relatively complicated systems and had some ambiguity in determining optimal feedback gains. A later suggestion involved employing modified constraint equations using the time integral of the constraint violations. ${ }^{13}$ Also, adaptive control and optimal control have been introduced ${ }^{14}$ to eliminate the ambiguity in feedback gains. A different approach ${ }^{15}$ to CVSM was also developed by using a penalty form of the constraint equations.

Conventional holonomic or nonholonomic constraints are defined as geometric constraints in this paper. The aforementioned works concentrate on geometric constraints. However, if the total energy of a dynamic system can be computed from the initial energy plus the time integral of the energy input rate due to external or internal forces, then the total energy can be artificially treated as a constraint. The violation of the total energy constraint due to numerical errors can be used as information to control these errors. It is a necessary condition for accurate simulation that both geometric and energy constraints be satisfied. When geometric constraint control is combined with energy constraint control, numerical simulation of a constrained dynamic system becomes more accurate.

First, constrained dynamic systems are introduced, using Lagrange multipliers, and then followed by the introduction of Baumgarte's CVSM. Conventional methods for implementing energy constraint control are then reviewed. An energy constraint control based on the gradient feedback of the energy constraint violation leads to a new method to control both geometric and energy constraint violations, socalled constraint violation stabilization using gradient feedback (CVSGF). A new convenient and effective method to implement energy constraint control in numerical simulation is developed based on the geometric interpretation of the relation between constraints in the phase space. Several combinations of energy constraint control with either Baumgarte's CVSM or the new CVSGF are also addressed. Finally, a new method for implementing constraint controls is developed by using the Euler method for integrating constraint control terms, even 
when higher-order integration methods are used for all other integrations.

\section{Constrained Dynamic Systems}

Constraints are either holonomic or nonholonomic. When the Lagrange multiplier method is applied to a dynamic system with holonomic constraints, ${ }^{4,5}$ the equations of motion are described by

$$
\begin{aligned}
M(q) \ddot{q}+\Phi_{q}^{T} \lambda & =G(q, \dot{q}, t) \\
\Phi(q, t) & =0
\end{aligned}
$$

where the holonomic constraint functions $\Phi: R^{n+1} \rightarrow R^{m}$, generalized coordinates $q \in R^{n}, m<n$, and time $t \geq 0$. In Eq. (1), $\lambda \in R^{m}$ is a Lagrange multiplier. The inertia matrix $M \in R^{n \times n}$ is positive definite, and $G \in R^{n}$ represents the remaining dynamic terms in the equation. Then the dynamic system with holonomic constraints is described by a set of $n$ differential equations (1) and $m$ algebraic equations (2).

If the Lagrange multiplier $\lambda$ can be computed or expressed in terms of $q, \dot{q}$, and $t$, then the system of algebraic differential equations can be solved numerically. A fundamental method for computing $\lambda$ without using implicit algorithms is to differentiate the constraint equation (2) twice with respect to time. This results in the equation

$$
\ddot{\Phi}(q, \dot{q}, \ddot{q}, t)=0
$$

or

$$
\Phi_{q} \ddot{q}+\left(\Phi_{q} \dot{q}\right)_{q} \dot{q}+2 \Phi_{q t} \dot{q}+\Phi_{t t}=0
$$

Since the inverse of the positive definite mass matrix $M$ exists, we can solve for $\ddot{q}$ in Eq. (1) to obtain

$$
\ddot{q}=-M^{-1} \Phi_{q}^{T} \lambda+M^{-1} G \equiv A(q, \dot{q}, \lambda, t)
$$

Equation (3b) can be rewritten as

$$
\Phi_{q} \ddot{q}-\gamma(q, \dot{q}, t)=0
$$

where $\gamma \equiv-\left(\Phi_{q} \dot{q}\right)_{q} \dot{q}-2 \Phi_{q t} \dot{q}-\Phi_{t t}$. Replacement of $\ddot{q}$ in Eq. (5) by Eq. (4) leads to

$$
-\Phi_{q} M^{-1} \Phi_{q}^{T} \lambda=-\Phi_{q} M^{-1} G+\gamma
$$

The $m \times m$ matrix $\Phi_{q} M^{-1} \Phi_{q}^{T}$ is nonsingular, since the $n \times n$ matrix $M$ is positive definite and the rank of $\Phi_{q}$ is $m$ where $m<n$. Thus, $\Phi_{q} M^{-1} \Phi_{q}^{T}$ can be inverted to obtain

$$
\lambda=\Lambda(q, \dot{q}, t)
$$

Finally, Eq. (7) is substituted back into Eq. (4) to yield

$$
\ddot{q}=A[q, \dot{q}, \Lambda(q, \dot{q}, t), t]
$$

Since the $n$ second-order differential equations in $q$ do not involve the $m$ Lagrange multipliers $\lambda$ in Eq. (8), the equations of motion can be solved numerically. In this paper, Eq. (8) is the differential equation that forms the basis for the numerical simulations.

\section{Baumgarte's Constraint Violation Stabilization Method}

A control $U(\Phi, \dot{\Phi}, t)$ can be added to the right side of Eq. (3a) in order to force reduction of the geometric constraint violations. Thus, we let

$$
\ddot{\Phi}=U(\Phi, \dot{\Phi}, t)
$$

Then Eq. (7) changes to

$$
\lambda=\Lambda[q, \dot{q}, U(q, \dot{q}, t), t]
$$

and Eq. (8) to

$$
\ddot{q}=A\{q, \dot{q}, \Lambda[q, \dot{q}, U(q, \dot{q}, t), t], t\}
$$

Equation (11) implies that the constrained dynamic system defined by Eqs. (1) and (2) is replaced by the system described by Eqs. (1) and (9) in order to effect geometric constraint control.

Since $\Phi$ is a function of $q$ and $t$, i.e., $\Phi(q, t)$, the modified constraint equation given by Eq. (9) has the form

$$
\ddot{\Phi}-U(\Phi, \dot{\Phi}, t) \equiv f_{n}(q, \dot{q}, \ddot{q}, t)=0
$$

Among many possible choices, Baumgarte suggested the control

$$
U \equiv-\alpha \dot{\Phi}-\beta \Phi
$$

Then Eq. (9) becomes

$$
\ddot{\Phi}+\alpha \dot{\Phi}+\beta \Phi=0
$$

In this case, the dynamic behavior of the new system is defined by Eqs. (1) and (13).

\section{Energy Constraint Control Methods}

There are at least two methods available in the literature for implementing an energy constraint using the Lagrange equations of motion. The first method is described by Baumgarte. ${ }^{12}$ The idea is to use the dynamic constraint equations modified from the original energy constraint equations in a manner similar to the nonholonomic case in Baumgarte's CVSM. If $\Psi$ is the energy constraint function, we let

$$
\dot{\Psi}+\eta \Psi=0, \quad \Psi=\Psi(q, \dot{q})
$$

or

$$
\Psi_{q} \dot{q}+\Psi_{\dot{q}} \ddot{q}+\eta \Psi=0
$$

where $\eta$ is a feedback gain constant. In general,

$$
\Psi \equiv T+V-(T+V)_{0}-\int_{t_{0}}^{t} \dot{E} \mathrm{~d} \tau
$$

where $\dot{E}$ is the energy input rate to the system. For a conservative system

$$
\Psi=E-E_{0}
$$

where $E$ is the total energy, expressed in terms of $q$ and $\dot{q}$. The Lagrange equation of motion for holonomic systems ${ }^{11}$ can be described by

$$
M(q) \ddot{q}+\Phi_{q}^{T}(q, t) \lambda=G(q, \dot{q}, t)
$$

If an energy constraint is added to the equation, then

$$
M \ddot{q}+\Phi_{q}^{T} \lambda+\omega_{\dot{q}}^{T} \mu=G
$$

where $\lambda$ and $\mu$ are Lagrange multipliers. Since the inertia matrix $M$ is positive definite, Eq. (2) can be written as

$$
\ddot{q}=M^{-1}\left(G-\Phi_{q}^{T} \lambda-\Psi_{q}^{T} \mu\right)
$$


Substituting Eq. (16) into Eq. (15), we obtain

$$
\Psi_{\dot{q}} M^{-1}\left(G-\Phi_{q}^{T} \lambda-\Psi_{\dot{q}}^{T} \mu\right)+\Psi_{q} \dot{q}+\eta \Psi=0
$$

or

$$
\left(\Psi_{\dot{q}} M^{-1} \Psi_{\dot{q}}^{T}\right) \mu=\Psi_{\dot{q}} M^{-1}\left(G-\Phi_{q}^{T} \lambda\right)+\Psi_{q} \dot{q}+\eta \Psi
$$

We note that $\Psi_{\dot{q}} M^{-1} \Psi_{\dot{q}}^{T}$ is invertible if $\Psi_{\dot{q}}$ is not identically zero. Then

$$
\mu=\left(\Psi_{\dot{q}} M^{-1} \Psi_{\dot{q}}^{T}\right)^{-1}\left[\Psi_{\dot{q}} M^{-1}\left(G-\Phi_{q}^{T} \lambda\right)+\Psi_{q} \dot{q}+\eta \Psi\right]
$$

Substituting Eq. (18) back into Eq. (16) results in the equation of motion without $\mu$, which is

$$
\begin{aligned}
\ddot{q} & =M^{-1}\left\{G-\Phi_{q}^{T} \lambda-\Psi_{\dot{q}}^{T}\left(\Psi_{\dot{q}} M^{-1} \Psi_{\dot{q}}^{T}\right)^{-1}\left[\Psi_{\dot{q}} M^{-1}\left(G-\Phi_{q}^{T} \lambda\right)\right.\right. \\
& \left.\left.+\Psi_{q} \dot{q}+\eta \Psi\right]\right\}
\end{aligned}
$$

Here $\eta$ should be chosen so that the energy constraint is stabilized.

The second method for implementing energy constraint control is based on the steepest descent algorithm. ${ }^{16}$ The correction forces are applied to the equations of motion so that the integration of $\dot{q}$ and $\ddot{q}$ moves in the direction that most rapidly reduces the violation of the energy constraint. ${ }^{17}$ To ensure that the minimum ${ }^{17,18}$ of $\Psi$ is zero, the negative gradient of $\Psi^{2}$ is fed back into the equation of motion (8). That is,

$$
\begin{aligned}
& \frac{\mathrm{d} q}{\mathrm{~d} t}=v-\rho_{q} \frac{\partial \Psi^{2}}{\partial q} \\
& \frac{\mathrm{d} v}{\mathrm{~d} t}=A(q, v, t)-\rho_{v} \frac{\partial \Psi^{2}}{\partial v}
\end{aligned}
$$

Here $\rho_{q}$ and $\rho_{\nu}$ are positive gain constants to be determined and, ideally, $\Psi(q, v)=0$. Since $\partial \Psi^{2} / \partial q=2 \Psi(\partial \Psi / \partial q)$ and $\partial \Psi^{2} /$ $\partial v=2 \Psi(\partial \Psi / \partial v)$, these control terms disappear when $\Psi=0$. In the analytic solution, $\Psi=0$ is satisfied. Thus, implementation of the energy constraint control in Eq. (20) does not change the exact solution of the original dynamic system equations. Both $\dot{q}$ and $v$ can be considered to represent the total time derivative of $q$. However, $\mathrm{d} / \mathrm{d} t$ is used to express the total time derivative when constraint control terms are added. The method in Eq. (20) of energy constraint control has been successfully applied in the computation of space and re-entry trajectories. ${ }^{19}$ Note that Eq. (20) is different from Eq. (19), and that Eq. (20) is simpler to implement.

Let us consider the relation between the foregoing two different energy constraint controls. The conventional Baumgarte's form is, from Eq. (14),

$$
\dot{\Psi}+\eta \Psi=0, \quad \Psi=\Psi(q, v)
$$

From Eq. (20) the energy constraint control in the form of gradient feedback is

$$
\begin{aligned}
& \frac{\mathrm{d} q}{\mathrm{~d} t}=v-2 \rho_{q} \Psi \frac{\partial \Psi}{\partial q} \\
& \frac{\mathrm{d} v}{\mathrm{~d} t}=A(q, v, t)-2 \rho_{\nu} \Psi \frac{\partial \Psi}{\partial v}
\end{aligned}
$$

Taking the time derivative of $\Psi$, we obtain

$$
\frac{\mathrm{d} \Psi}{\mathrm{d} t}=\frac{\partial \Psi}{\partial q} \frac{\mathrm{d} q}{\mathrm{~d} t}+\frac{\partial \Psi}{\partial \nu} \frac{\mathrm{d} v}{\mathrm{~d} t}
$$

Substitution of the expression in Eq. (22) for $\mathrm{d} q / \mathrm{d} t$ into Eq. (23) results in

$$
\frac{\mathrm{d} \Psi}{\mathrm{d} t}=(\dot{\Psi})_{\text {chain }}-2 \Psi\left[\rho_{q}\left(\frac{\partial \Psi}{\partial q}\right)\left(\frac{\partial \Psi}{\partial q}\right)^{T}+\rho_{\nu}\left(\frac{\partial \Psi}{\partial v}\right)\left(\frac{\partial \Psi}{\partial v}\right)^{T}\right]
$$

where

$$
(\dot{\Psi})_{\text {chain }} \equiv \frac{\partial \Psi}{\partial q} v+\frac{\partial \Psi}{\partial v} A
$$

The constraint control terms in Eq. (22) make $d \Psi / d t$ in Eq. (23) different from $(\dot{\Psi})_{\text {chain }}$ in Eq. (25), which is obtained from the chain rule of differential calculus. For small enough integration step size $h,(\dot{\Psi})_{\text {chain }}$ can be assumed to be very small compared with the other two terms on the right side of Eq. (24) by choosing gains $\rho_{q}$ and $\rho_{v}$ of order $h^{-1}$. A more detailed discussion of this assumption is given in the next section. Then Eq. (24) becomes

$$
\frac{\mathrm{d} \Psi}{\mathrm{d} t}=-\alpha_{e} \Psi
$$

where

$$
\alpha_{e}=2\left[\rho_{q}\left(\frac{\partial \Psi}{\partial q}\right)\left(\frac{\partial \Psi}{\partial q}\right)^{T}+\rho_{\nu}\left(\frac{\partial \Psi}{\partial v}\right)\left(\frac{\partial \Psi}{\partial v}\right)^{T}\right]
$$

Note that Eqs. (21) and (26) have the same form. But Eq. (22), which leads to Eq. (26), is much easier to implement than Eq. (21), which results in Eq. (19).

\section{Constraint Violation Stabilization Using Gradient Feedback}

The gradient concept behind the steepest descent algorithm can also be applied to conventional geometric (holonomic) constraints. The correction is applied to the equations of motion so that the integration of $\dot{q}$ and $\ddot{q}$ moves in the direction that results in the most rapid reduction of the geometric constraint violation. ${ }^{18}$ Since a holonomic constraint is not a function of $\dot{q}$, the gradient feedback control is applied only to the $\mathrm{d} q / \mathrm{d} t$ equation in the formulation of Eq. (8). That is,

$$
\begin{aligned}
& \frac{\mathrm{d} q}{\mathrm{~d} t}=v-\sum_{i=1}^{m} \zeta_{i} \frac{\partial \Phi_{i}^{2}}{\partial q} \\
& \frac{\mathrm{d} v}{\mathrm{~d} t}=A(q, v, t)
\end{aligned}
$$

where each $\zeta_{i}$ represents a gain to be determined. Again note that $\dot{q}$ is replaced by the symbol $v$. Both $v$ and $\dot{q}$ represent the total time derivative of $q$, whereas $\mathrm{d} q / \mathrm{d} t$ represents a total time derivative to be integrated numerically. The use of $\Phi_{i}^{2}$ rather than $\Phi_{i}$ in the gradient-based control results in $\Phi_{i}=0$ as the minimum error. In this case, the feedback control becomes smaller as $\Phi_{i}$ approaches zero, which is also desirable. This also means that the control term disappears when $\Phi=0$, since $\partial \Phi^{2} / \partial q=2 \Phi(\partial \Phi / \partial q)$. Note that $\Phi=0$ is satisfied continuously in the exact solution, which implies that the nature of the exact solution of the original dynamic system is not altered by the introduction of the constraint control in CVSGF.

It is possible to show how Eq. (28), which is the method of CVSFG, is related to Baumgarte's conventional CVSM, which is based on

$$
\ddot{\Phi}+\alpha \dot{\Phi}+\beta \Phi=0
$$

First we take the time derivative of $\Phi_{i}(q, t)$ to obtain

$$
\frac{\mathrm{d} \Phi_{i}}{\mathrm{~d} t}=\frac{\partial \Phi_{i}}{\partial q} \frac{\mathrm{d} q}{\mathrm{~d} t}+\frac{\partial \Phi_{i}}{\partial t}, \quad i=1,2, \ldots, m
$$


Substitution of the expression in Eq. (28) for $\mathrm{d} q / \mathrm{d} t$ into Eq. (30) results in

$$
\frac{\mathrm{d} \Phi_{i}}{\mathrm{~d} t}=\left(\dot{\Phi}_{i}\right)_{\text {chain }}-2\left(\frac{\partial \Phi_{i}}{\partial q}\right) \sum_{j=1}^{m} \zeta_{j} \Phi_{j}\left(\frac{\partial \Phi_{j}}{\partial q}\right)^{T}
$$

where $\partial \Phi_{i} / \partial q \in R^{1 \times n}$ and

$$
\left(\dot{\Phi}_{i}\right)_{\text {chain }} \equiv \frac{\partial \Phi_{i}}{\partial q} v+\frac{\partial \Phi_{i}}{\partial t}
$$

The control term in the first equation in Eq. (28) makes $\mathrm{d} \Phi_{i} / \mathrm{d} t$ in Eq. (30) different from $\left(\dot{\Phi}_{i}\right)_{\text {chain }}$ in Eq. (32), which results from the chain rule of differential calculus. Assume that we use a numerical integration algorithm of order $N$. Then $\Phi_{k}$ will be of order $h^{N}$, where $h$ is the integration step size. The errors in $v, \partial \Phi_{i} / \partial q$, and $\partial \Phi_{i} / \partial t$ are also of order $h^{N}$. If $v^{*},\left(\partial \Phi_{i} / \partial q\right)^{*}$, and $\left(\partial \Phi_{i} / \partial t\right)^{*}$ are the exact values of $v, \partial \Phi_{i} / \partial q$, and $\partial \Phi_{i} / \partial t$, respectively, and their fractional numerical errors are represented by $\epsilon, \delta_{q}$, and $\delta_{t}$, then it follows that

$$
\begin{gathered}
v=v^{*}\left[1+\epsilon\left(h^{N}\right)\right] \\
\frac{\partial \Phi_{i}}{\partial t}=\left(\frac{\partial \Phi_{i}}{\partial t}\right)^{*}\left[1+\delta_{t}\left(h^{N}\right)\right] \\
\frac{\partial \Phi_{i}}{\partial q}=\left(\frac{\partial \Phi_{i}}{\partial q}\right)^{*}\left[1+\delta_{q}\left(h^{N}\right)\right]
\end{gathered}
$$

Then

$$
\left(\dot{\Phi}_{i}\right)_{\text {chain }} \approx\left(\frac{\partial \Phi_{i}}{\partial q}\right)^{*} v^{*}\left(1+\delta_{q}+\epsilon\right)+\left(\frac{\partial \Phi_{i}}{\partial t}\right)^{*}\left(1+\delta_{t}\right)
$$

Note that $\left(\partial \Phi_{i} / \partial q\right)^{*} v^{*}+\left(\partial \Phi_{i} / \partial t\right)^{*}=\dot{\Phi}_{i}^{*}=0$, even though $v^{*}$, $\left(\partial \Phi_{i} / \partial q\right)^{*}$, and $\left(\partial \Phi_{i} / \partial t\right)^{*}$ are each of order $h^{0}$. Then Eq. (36) becomes

$$
\left(\dot{\Phi}_{i}\right)_{\text {chain }} \approx\left(\frac{\partial \Phi_{i}}{\partial q}\right)^{*} v^{*}\left(\delta_{q}+\epsilon\right)+\left(\frac{\partial \Phi_{i}}{\partial t}\right)^{*} \delta_{t}
$$

which is of order $h^{N}$. Next, we consider the last term $\left(\partial \Phi_{i} /\right.$ $\partial q) \sum \zeta_{j} \Phi_{j}\left(\partial \Phi_{i} / \partial q\right)^{T}$ of Eq. (31). The gain constant $\zeta_{j}$ can be chosen to be of order $1 / h$ since the numerical stability depends on the value of $\lambda_{\Phi} h$, and the eigenvalue $\lambda_{\Phi}$ is proportional to $\zeta_{j}$ for this first-order system. We recall that $\left(\partial \Phi_{i} / \partial q\right)$ is of order $h^{0}$ and $\Phi_{i}$ is of order $h^{N}$. Therefore, the second term on the right side of Eq. (31) is of order $h^{N-1}$. In this case, $\left(\dot{\Phi}_{i}\right)_{\text {chain }}$ in Eq. (31) can be neglected for small enough $h$, and Eq. (31) becomes

$$
\frac{\mathrm{d} \Phi_{i}}{\mathrm{~d} t} \cong-2\left(\frac{\partial \Phi_{i}}{\partial q}\right) \sum_{j=1}^{m} \zeta_{j} \Phi_{j}\left(\frac{\partial \Phi_{j}}{\partial q}\right)^{T}, \quad i=1,2, \ldots, m
$$

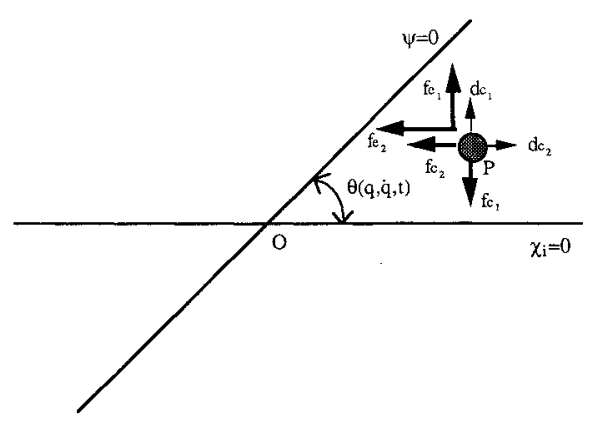

Fig. 1 Two-dimensional geometric interpretation of CVSMs combined with energy constraint control in phase space.
Equation (38) then becomes

$$
\frac{\mathrm{d} \Phi}{\mathrm{d} t}=-\alpha_{g} \Phi
$$

where

$$
\alpha_{g}=2\left(\frac{\partial \Phi}{\partial q}\right)\left(\frac{\partial \Phi}{\partial q}\right)^{T} \zeta
$$

and $\zeta$ represents an $m \times m$ diagonal matrix.

\section{New Method of Implementing \\ Energy Constraint Control}

In the $q, \dot{q}$ phase space, the surfaces of constant energy and for conventional holonomic or nonholonomic constraints are not, in general, perpendicular to each other. The surfaces are given by

$$
\Psi(q, \dot{q})=0 \text { : energy constraint }
$$

$$
\chi_{i}(q, \dot{q}, t)=0, \quad i=1,2, \ldots, m: \text { geometric constraints }
$$

In general,

$$
\left[\frac{\partial \Psi}{\partial\left(\begin{array}{c}
q \\
\dot{q}
\end{array}\right)}\right]\left[\frac{\partial \chi_{i}}{\partial\left(\begin{array}{c}
q \\
\dot{q}
\end{array}\right)}\right]^{T} \neq 0, \quad i=1,2, \ldots, m
$$

where $q, \dot{q} \in R^{n}, \Psi: R^{2 n} \rightarrow R$, and $\chi_{i}: R^{2 n+1} \rightarrow R$. One case where the energy and geometric constraint surfaces are orthogonal is illustrated in the following example. Let

$$
\Psi(\dot{q})=0
$$

and

$$
\chi_{i}(q, t)=0, \quad i=1,2, \ldots, m
$$

Then

$$
\begin{aligned}
& {\left[\frac{\partial \Psi}{\partial\left(\begin{array}{c}
q \\
\dot{q}
\end{array}\right)}\right]^{T}=\left[\begin{array}{c}
0 \\
\frac{\partial \Psi}{\partial \dot{q}}
\end{array}\right]} \\
& {\left[\frac{\partial \chi_{i}}{\partial\left(\begin{array}{l}
q \\
\dot{q}
\end{array}\right)}\right]^{T}=\left[\begin{array}{c}
\frac{\partial \chi_{i}}{\partial q} \\
0
\end{array}\right]}
\end{aligned}
$$

Thus,

$$
\left[\frac{\partial \Psi}{\partial\left(\begin{array}{l}
q \\
\dot{q}
\end{array}\right)}\right]\left[\frac{\partial \chi_{i}}{\partial\left(\begin{array}{l}
q \\
q
\end{array}\right)}\right]^{T}=0
$$

The solution to the equations of motion of a given system can be interpreted as a point moving in the state-variable phase space. The necessary condition for the exact simulation of the given equations of motion is that the point moves along the common intersection of all the constraint surfaces in the phase space. Numerical errors in the simulation represent disturbances that continuously perturb the point from this common intersection. The constraint violation control is designed to 
minimize the effect of these disturbances and keep the point close to the constraint-surface intersection.

If violations of both geometric and energy constraints are relatively small, then the point is always close to the constraint surface intersection. If a two-dimensional view is taken in the neighborhood of the intersection, then the lines indicating the constraints can be viewed as nearly linear, as in Fig. 1. The intersection in the phase space is the origin $O$ in this twodimensional view, and the phase point is denoted as $P$ in the figure. The point $P$ is continually disturbed by the numerical integration errors during the simulation. The disturbance can be considered to be composed of two orthogonal components $\left(d_{c_{1}}, d_{c_{2}}\right)$ in the plane. When CVSM is applied to control the geometric constraint violation, CVSM forces the point $P$ to move toward the $\chi_{i}=0$ axis. Thus, the control of CVSM generally has two components $\left(f_{c_{1}}, f_{c_{2}}\right)$, where $f_{c_{1}}$ is always directed toward the $\chi_{i}=0$ axis. Note that the directions of $d_{c_{1}}$, $d_{c_{2}}$, and $f_{c_{2}}$ may be reversed in the figure, and that the relative angle $\theta$ between two axes can be a function of the state variables and time, i.e., $\theta=\theta(q, \dot{q}, t)$.

If the constraint surfaces of the geometric and energy constraints are not orthogonal to each other in the phase space, which, in general, will be the case, then the relative position of $P$ with respect to the energy constraint surface is changed by applying geometric constraint control. There exists no guarantee that $P$ moves toward the intersection $O$ of the two constraint surfaces. In many cases, $P$ moves farther from the origin $O$ when applying geometric constraint control alone. This phenomenon can be noted later in the test simulations. When strong geometric constraint control is applied to ensure very small geometric constraint errors, the resulting drift in the total energy can in many cases become very large. This, in turn, causes large errors in the state variables as functions of time. These arguments explain why a combination of geometric and energy constraint controls is essential for accurate simulation, i.e., to keep the point $P$ close to the exact solution point $O$ in Fig. 1 .

The conventional methods described in the previous section for implementing energy constraint control require the point to move toward the $\Psi=0$ axis. The control can be considered to be composed of two orthogonal components $\left(f_{e_{1}}, f_{e_{2}}\right)$, one perpendicular and one parallel to the $\chi_{i}=0$ axis. CVSM forces the point $P$ to move toward the $\chi_{i}=0$ axis, while the energy control makes the point $P$ move toward the $\Psi=0$ axis. Note again that the relative angle $\theta$ is a function of state variables and time, which makes the direction of the sum of $\left(f_{e_{1}}, f_{e_{2}}\right)$ difficult to predict. If CVSMs are combined with conventional energy methods, the two different controls are geometrically coupled. That is, the control to make $P$ move toward the origin $O$ may not be simple.

A solution to this difficulty for the case of holonomic systems is to make the control of the energy constraint parallel to the geometric constriant. That is, in effect we have

$$
\left[\frac{\partial \Psi}{\partial\left(\begin{array}{c}
q \\
\dot{q}
\end{array}\right)}\right]^{T}=\left[\begin{array}{c}
0 \\
\frac{\partial \Psi}{\partial \dot{q}}
\end{array}\right]
$$

by setting $\rho_{q}=0$. With $\rho_{q}=0$, Eq. (20) becomes

$$
\begin{aligned}
& \frac{\mathrm{d} q}{\mathrm{~d} t}=v \\
& \frac{\mathrm{d} v}{\mathrm{~d} t} \equiv A(q, v, t)-\rho_{v} \frac{\partial \Psi^{2}}{\partial v}
\end{aligned}
$$

Note that the geometric interpretation is not changed by replacing $\Psi$ with $\Psi^{2}$. Replacement of $\Psi$ by $\Psi^{2}$ makes the effect of the feedback control on the point $P$ proportionally less as the point moves closer to the origin. That is,

$$
\rho_{v} \frac{\partial \Psi^{2}}{\partial v}=2 \rho_{v} \Psi \frac{\partial \Psi}{\partial v}
$$

and the energy correction term in Eq. (44) varies linearly with $\Psi$. By using Eq. (44) rather than Eq. (20), the control on the energy constraint becomes parallel to the geometric constraint, i.e., $f_{e_{1}}=0$ and $\rho_{q}=0$. Then

$$
\left[\frac{\partial \chi_{i}}{\partial\left(\begin{array}{l}
q \\
\dot{q}
\end{array}\right)}\right]^{T}=\left[\begin{array}{c}
\frac{\partial \chi_{i}}{\partial q} \\
0
\end{array}\right]
$$

and, from Eqs. (43) and (45),

$$
\left[\frac{\partial \Psi}{\partial\left(\begin{array}{l}
q \\
\dot{q}
\end{array}\right)}\right]\left[\frac{\partial \chi_{i}}{\partial\left(\begin{array}{l}
q \\
\dot{q}
\end{array}\right)}\right]^{T}=0, \quad i=1,2, \ldots, m
$$

Application of the control on the geometric constraint forces the point to move toward the geometric constraint. However, it also changes the relative position of the point with respect to the energy constraint, since the two different surfaces are not perpendicular to each other. Because the control on the energy constraint is parallel to the geometric constraints, the energy control does not change the relative position of the point $P$ with respect to the $\chi_{i}=0$ axis. Thus, the $f_{e_{1}}$ component has been removed by setting $\rho_{q}=0$. Appropriate choice of the gains in Eq. (44) will force the point $P$ to move toward the origin $O$ despite the presence of truncation errors.

The difficulty associated with the proper choice of these gains is dependent on how the constraints are coupled in phase space. The variable $Y_{c i}(q, \dot{q}, t)$, defined as

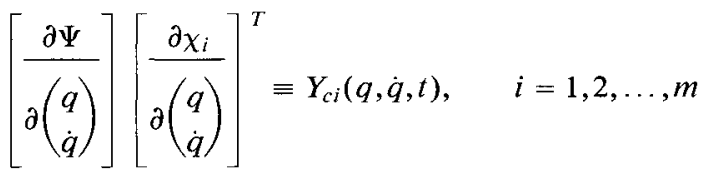

can be used as a measure of the degree of coupling when an energy constraint control and geometric constraint controls are combined. The large the magnitude of $Y_{c i}$, the more substantial the coupling.

The aforementioned energy constraint controls can be utilized for more accurate simulation in the case where the exact total energy of a dynamic system at each time frame during integration is known, even though the given system is nonconservative. However, it is not easy to derive and compute the total energy in complex dynamic systems.

\section{Combination of Geometric Constraint Control and Energy Constraint Control}

Baumgarte's CVSM in Eq. (11) with $U=-\alpha \dot{\Phi}-\beta \Phi$ leads to a set of equations of the form

$$
\begin{aligned}
& \frac{\mathrm{d} q}{\mathrm{~d} t}=v \\
& \frac{\mathrm{d} v}{\mathrm{~d} t}=A_{B}(q, v, t, \alpha, \beta)
\end{aligned}
$$


On the other hand, when CVSGF in Eq. (28) is used for geometric constraint control, the equations are

$$
\begin{aligned}
\frac{\mathrm{d} q}{\mathrm{~d} t} & =v-\sum_{i=1}^{m} \zeta_{i} \frac{\partial \Phi_{i}^{2}}{\partial q} \\
\frac{\mathrm{d} v}{\mathrm{~d} t} & =A(q, v, t)
\end{aligned}
$$

where $\zeta_{i}$ is a gain to be determined. Note again that $\dot{q}$ is replaced by $v$ and that $A_{B}=A$ if $\alpha=\beta=0$. Both $v$ and $\dot{q}$ represent the total time derivative of $q$. In this paper, $\mathrm{d} / \mathrm{d} t$ is used to express the time derivative to be integrated numerically in the state equations. Equation (48) is quite different in form from Eq. (49), even though both equations share the same philosophy, i.e., that feedback control is applied to stabilize geometric constraint violations. These equations can be combined with the energy constraint controls. If Eq. (20) is combined with Eq. (49), then

$$
\begin{aligned}
& \frac{\mathrm{d} q}{\mathrm{~d} t}=v-\sum_{i=1}^{m} \zeta_{i} \frac{\partial \Phi_{i}^{2}}{\partial q}-\rho_{q} \frac{\partial \Psi^{2}}{\partial q} \\
& \frac{\mathrm{d} v}{\mathrm{~d} t}=A(q, v, t)-\rho_{\nu} \frac{\partial \Psi^{2}}{\partial v}
\end{aligned}
$$

For the case where $\rho_{q}=0$ as in Eq. (44),

$$
\begin{aligned}
& \frac{\mathrm{d} q}{\mathrm{~d} t}=v-\sum_{i=1}^{m} \zeta_{i} \frac{\partial \Phi_{i}^{2}}{\partial q} \\
& \frac{\mathrm{d} v}{\mathrm{~d} t}=A(q, v, t)-\rho_{v} \frac{\partial \Psi^{2}}{\partial v}
\end{aligned}
$$

Combination of Eqs. (20) and (48) results in

$$
\begin{aligned}
& \frac{\mathrm{d} q}{\mathrm{~d} t}=v-\rho_{q} \frac{\partial \Psi^{2}}{\partial q} \\
& \frac{\mathrm{d} v}{\mathrm{~d} t}=A_{B}(q, v, t, \alpha, \beta)-\rho_{v} \frac{\partial \Psi^{2}}{\partial v}
\end{aligned}
$$

Finally, if Eq. (44) is combined with Eq. (48), then

$$
\begin{aligned}
& \frac{\mathrm{d} q}{\mathrm{~d} t}=v \\
& \frac{\mathrm{d} v}{\mathrm{~d} t}=A_{B}(q, v, t, \alpha, \beta)-\rho_{v} \frac{\partial \Psi^{2}}{\partial v}
\end{aligned}
$$

The geometric interpretation of these four equations is similar to the interpretation in the previous section. Note that the vector associated with $\partial \Phi_{i}^{2} / \partial q$ in Eq. (51) is orthogonal to the $\Phi_{i}=0$ surface since it has the direction of the gradient. On the other hand, the vector associated with $\partial \Psi^{2} / \partial v$ in Eq. (51) is orthogonal to each previous vector in the sense of satisfying Eq. (46) with $\Phi_{i} \equiv \chi_{i}$. Thus, the choice of Eq. (51) for constraint feedback control decouples the geometric and energy constraint controls. This means that the gains $\zeta_{i}$ and $\rho_{v}$ in Eq. (51) can be chosen separately based on Eqs. (27) and (40) with $\rho_{q}=0$. This represents a distinct advantage of CVSGF over Baumgarte's CVSM when combined with energy constraint control, since now the coupling as measured by $Y_{c i}$ in Eq. (47) does not exist.

\section{Constraint Control Using Euler Integration}

When a multipass integration algorithm such as RungeKutta fourth-order (RK-4) is used in the simulation of a dynamic system with Baumgarte's modified constraint equations, it is not clear whether the constraint controls should be applied in every pass or not. In most test simulations, using the controls in every pass resulted in divergence of the constraint violations. In a multipass integration algorithm, it is therefore vital to determine which pass or passes to use for the application of the constraint control. This problem is automatically solved when we utilize the single-pass Euler method for integrating the constraint control terms, as explained in this section.

Consider a differential equation $\dot{x}=f(x, t)$ and assume that a constraint control $u(x, t)$ is incorporated into the state equation. Then the modified equation can be written as

$$
\dot{x}=\bar{f}[x, u(x, t), t]
$$

The right side of the equation can be split into two terms in the following way:

$$
\dot{x}=\bar{f}[x, u(x, t), t] \equiv f(x, t)+\bar{U}(u, x, t)
$$

Here $\bar{U}(u, x, t)$ is the term containing the constraint control. For the conventional integration algorithms such as Euler, Adams-Bashforth second-order (AB-2), and third-order (AB3 ), the numerical integration of $\mathrm{Eq}$. (55) uses the following difference equations:

$$
x_{k+1}=x_{k}+h \hat{f}_{k}
$$

where

$$
\begin{array}{rlrl}
\hat{f}_{k} & =\bar{f}_{k} & \text { for Euler } \\
& =\frac{\left(3 \bar{f}_{k}-\bar{f}_{k-1}\right)}{2} & & \text { for } \mathrm{AB}-2 \\
& =\frac{\left(23 \bar{f}_{k}-16 \bar{f}_{k-1}+5 \bar{f}_{k-2}\right)}{12} & & \text { for } \mathrm{AB}-3
\end{array}
$$

In integrating $\dot{x}$ numerically to obtain $x$, an alternative method is to use one integration method to integrate the term $f(x, t)$ and a second method, the Euler method, to integrate the constraint control term $\bar{U}(u, x, t)$. The reasoning behind this alternative method is that accurate integration of the constraint control term $\bar{U}(u, x, t)$ is relatively unimportant, since this term is only applying a correction to drive the constraint violation toward zero. For this purpose, simple Euler integration, which has a favorable stability region in the $\lambda h$ plane, is just as effective as a higher-order integration algorithm. When the forward Euler method for constraint control integration is combined with Euler, AB-2, or AB-3 integration, the difference equations become

$$
\begin{gathered}
\hat{x}_{k+1}=x_{k}+h \hat{f}_{k} \\
x_{k+1}=\hat{x}_{k+1}+h \bar{U}\left[u\left(x_{k}, t_{k}\right), x_{k}, t_{k}\right]
\end{gathered}
$$

where

$$
\begin{aligned}
\hat{f}_{k} & =f_{k} & & \text { for Euler } \\
& =\frac{\left(3 f_{k}-f_{k-1}\right)}{2} & & \text { for } \mathrm{AB}-2 \\
& =\frac{\left(23 f_{k}-16 f_{k-1}+5 f_{k-2}\right)}{12} & & \text { for } \mathrm{AB}-3
\end{aligned}
$$

Note that $\hat{x}_{k+1}$ in Eq. (57) represents the integrated result before the constraint control is applied, whereas $x_{k+1}$ in Eq. (58) is the integrated result after application of the constraint control.

Alternatively, backward Euler integration can be used in place of the forward Euler integration represented by Eq. (58). In this case, Eq. (58) is replaced by

$$
x_{k+1}=\hat{x}_{k+1}+h \bar{U}\left[u\left(\hat{x}_{k+1}, t_{k+1}\right), \hat{x}_{k+1}, t_{k+1}\right]
$$




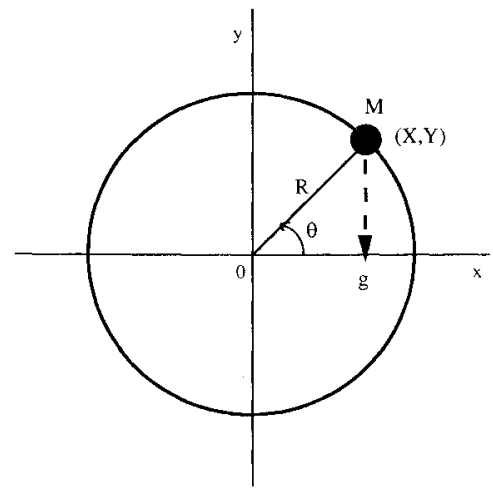

Fig. 2 Unit mass rotating on a unit circle.

Here the constraint control uses the constraint violation based on $\hat{x}_{k+1}$ rather than $x_{k}$.

\section{Test Simulations}

It will be shown experimentally why control of geometric constraints only cannot yield accurate values of the state variables in the numerical simulation of constrained dynamic systems, even if the control may achieve successful suppression of the geometric constraint violations. The energy constraint control will turn out to be necessary for accurate simulation even when independent coordinates are chosen in the numerical simulation. The new CVSGF will be also compared with conventional methods and examined with the following example.

Example 1: A unit mass moves along a unit circle in the $X Y$ plane (see Fig. 2). A gravity force $M g(M=1)$ is applied in the negative $Y$ direction. This is just a simple pendulum problem, with the position of the unit pendulum mass with respect to the suspension point represented by the dependent rectangular coordinates $X$ and $Y$, rather than the usual independent polar coordinate $\theta$. The pendulum length equals unity in the example. The equations of motion without constraint control are

$$
\begin{gathered}
\frac{\mathrm{d}}{\mathrm{d} t} X=V_{X} \\
\frac{\mathrm{d}}{\mathrm{d} t} Y=V_{Y} \\
\frac{\mathrm{d}}{\mathrm{d} t} V_{X}=-X \frac{V_{X}^{2}+V_{Y}^{2}-g Y}{X^{2}+Y^{2}} \\
\frac{\mathrm{d}}{\mathrm{d} t} V_{Y}=-Y \frac{V_{X}^{2}+V_{Y}^{2}-g Y}{X^{2}+Y^{2}}
\end{gathered}
$$

The gravity $g$ is fixed at $\mathbf{1}$ in the test simulations. In terms of rectangular coordinates $X$ and $Y$, the holonomic constraint equation is

$$
\Phi=1 / 2\left(X^{2}+Y^{2}-1\right)=0
$$

The energy constraint is

$$
\Psi=T+V-\left(T_{0}+V_{0}\right)=1 / 2\left(\dot{X}^{2}+\dot{Y}^{2}\right)+g Y-\left(T_{0}+V_{0}\right)=0
$$

The initial conditions are

$$
X(0)=1, \quad Y(0)=0, \quad \dot{X}(0)=0, \quad \dot{Y}(0)=2
$$

With the initial conditions, the pendulum starts with sufficient upward velocity to cause it to rotate continuously but with periodically varying angular velocity.
Adams-Bashforth third (AB-3) integration method is used with the integration step size $h=0.01 \mathrm{~s}$ in the test simulations. The integration step size is small enough to choose the gain constants in the new CVSGF based on the relations in Eqs. (27) and (40). The startup problem of AB-3 is resolved by using Runge-Kutta fourth-order (RK-4) integration to compute necessary initial startup conditions. With this simple example, six different cases are compared in geometric constraint violation $\Phi$, energy constraint violation $\Psi$, and time history of the errors in the state variable $X$. The case where no constraint controls are applied is shown in Figs. 3-5, as well as the case where Baumgarte's geometric constraint control is applied. In Baumgarte's CVSM, critical damping is used for the gains $\alpha$ and $\beta$, i.e., $\beta=\alpha^{2} / 4$, and $\beta=40$ is chosen to yield $|\lambda| h=0.0632$ for the eigenvalue $\lambda$ of the modified constraint equation (29) and integration step size $h=0.01$. If gain constants larger than $\beta=40$ are employed, smaller constraint violations can be obtained. However, state-variable errors become much larger than those in Fig. 5. The case where Baumgarte's geometric constraint control is combined with the energy constraint control, i.e., Eq. (53), is also compared in the figures along with the new CVSGF in the form of Eq. (51). Note that the Euler constraint control in Eqs. (57) and (58) is combined with Eq. (51). In these cases, the gain parameters $\rho$ and $\zeta$ are determined in the following way: Substituting the expressions for $\Phi$ and $\Psi$ in Eqs. (61) and (62) into Eqs. (27) and (40), we obtain the following formulas for the eigenvalues $-\alpha_{g}$ and $-\alpha_{e}$ in the first-order equation obeyed approximately by the geometric and energy constraints:

$$
\begin{gathered}
\alpha_{g}=2 \zeta\left(X^{2}+Y^{2}\right) \approx 2 \zeta \\
\alpha_{e}=2 \rho\left(\dot{X}^{2}+\dot{Y}^{2}\right) \approx 4 \rho\left(E_{0}-g Y\right)
\end{gathered}
$$

Since $\Phi \approx 0$ and $\Psi \approx 0$ in accurate simulations, such as those represented in Figs. 3-5, we have assumed in writing Eqs. (64) and (65) that

$$
X^{2}+Y^{2}=1
$$

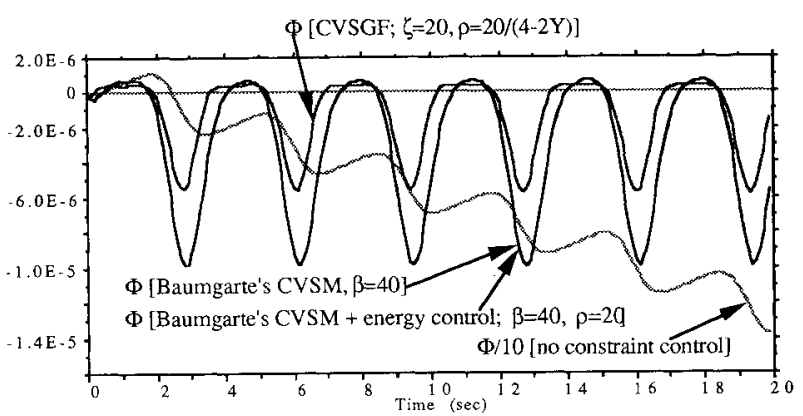

Fig. 3 Example $1[\mathrm{AB}-3, h=0.01]$ : geometric constraint violations.

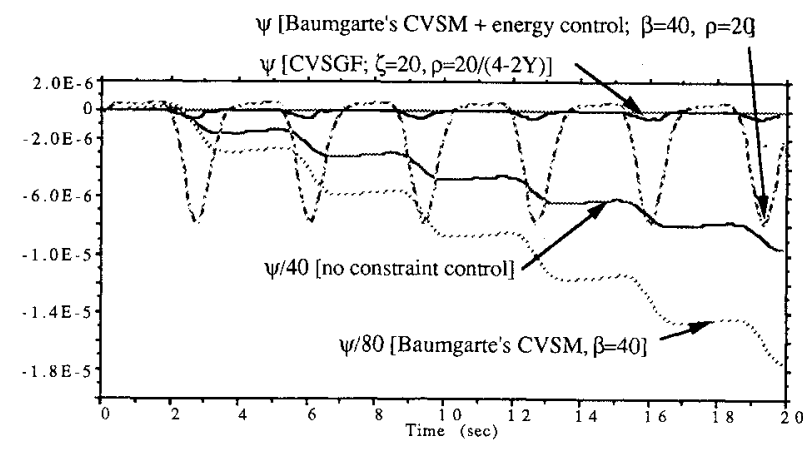

Fig. 4 Example $1[\mathrm{AB}-3, h=0.01]$ : energy constraint violations. 


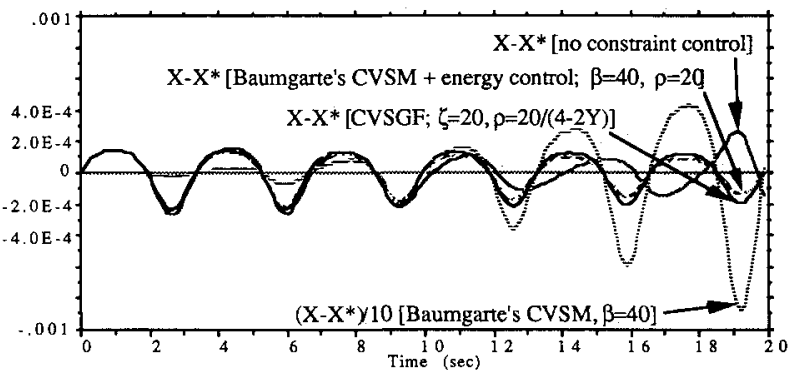

Fig. 5 Example 1 [AB-3, $h=0.01$ ]: errors in the state variable $X$.

and

$$
\dot{X}^{2}+\dot{Y}^{2}=2\left(E_{0}-g Y\right)
$$

Here $\alpha_{g}$ and $\alpha_{e}$ are the characteristic roots of the first-order differential equations (26) and (39) that describe the approximate dynamic behavior of the constraints $\Phi$ and $\Psi$. Also, $\alpha_{g}=\alpha_{e}=40$ is chosen in the figures and corresponds to $\lambda h=0.0632$, as in Baumgarte's geometric constraint control.

If an independent coordinate $\theta$ is chosen as a state variable, where $\theta$ is a counterclockwise angular displacement of the unit mass from the positive $X$ axis, then the equation of motion is simply

$$
\ddot{\theta}+\cos \theta=0
$$

and the exact solution is given in Ref. 5 .

The foregoing example is relatively simple, but complex enough to demonstrate the nature of numerical difficulties in simulating general dynamic systems. Since the exact solution is available, the simulation accuracies of the various constraint control methods can be vividly compared. More complex examples are simulated in Ref. 19.

Figure 3 shows that the geometric constraint controls, in either Baumgarte's CVSM or the new CVSGF, make the geometric constraint violations stable. Note that the case of no constraint control is divided by $\mathbf{1 0}$ for comparison in the figure. That is, the actual constraint violation is 10 times larger than the one in the figure. The logarithmic scale is not used since the tendency of constraint violations in time is considered to be more crucial than the amount of violations in understanding the nature of violations in most cases. Figure $4 \mathrm{com}-$ pares energy constraint violations $\Psi$. Note that some time histories are divided by corresponding numbers for comparison in the figure. It shows that Baumgarte's geometric constraint control yields the largest energy constraint violation without energy constraint control. Finally, in Fig. 5 the time domain errors in $X$ are compared. The reference solution for $X^{*}$ is obtained by using RK-4 and $h=0.00001$ to integrate Eq. (68). Figure 5 shows that Baumgarte's CVSM with energy constraint control cannot be directly compared with the new CVSGF since it is not clear how to choose optimal gain constants in each method. However, with the new CVSGF, it is easier to understand the nature of the constraint controls, as in the previous geometric analysis in the phase space.

\section{Conclusion}

In numerical simulation of constrained dynamic systems, only geometric constraint violation has been considered to examine the accuracy of simulation, which led to several geometric constraint control methods. However, this paper shows that geometric constraint control without energy constraint control worsens the time domain errors in state variables. The new constraint violation stabilization using gradient feedback is very effective and easy to implement in controlling errors of either geometric constraints or an energy constraint. This method can be reinforced even by an Euler constraint control. The relation between constraint controls and violations is also geometrically understood in the phase space, which leads to the most effective combination of both geometric and energy constraint controls.

\section{References}

${ }^{1}$ Hooker, W., and Margulies, G., "The Dynamical Attitude Equations for an $n$-Body Satellite," Journal of Astronautical Science, Vol. 12, No. 4, 1965, pp. 123-128.

${ }^{2}$ Roberson, R., "A Form of the Translational Dynamical Equations for Relative Motion in Systems of Many Non-Rigid Bodies," Acta Mechanica, Vol. 14, 1972, pp. 297-308.

${ }^{3}$ Kane, T., and Levinson, D., "Formulation of Equations of Motion for Complex Spacecraft," Journal of Guidance and Control, Vol. 3, No. 2, 1980, pp. 99-112.

${ }^{4}$ Greenwood, D. T., Principles of Dynamics, Prentice-Hall, Englewood Cliffs, NJ, 1988.

${ }^{5}$ Greenwood, D. T., Classical Dynamics, Prentice-Hall, Englewood Cliffs, NJ, 1977.

${ }^{6}$ Gear, C. W., "The Simultaneous Numerical Solution of Differential-Algebraic Equations," IEEE Transactions on Circuit Theory, Vol. CT-18, No. 1, 1971, pp. 89-95.

${ }^{7}$ Petzold, L. R., "Differential/Algebraic Equations Are Not ODE's," SIAM Journal of Scientific and Statistical Computing, Vol. 3, No. 3, 1982, pp. 367-384.

${ }^{8}$ Gear, C. W., "Differential-Algebraic Equations," Computer Aided Analysis and Optimization of Mechanical System Dynamics, edited by E. J. Haug, NATO ASI Series, Series F, Vol, 9, SpringerVerlag, Heidelberg, 1984, pp. 323-334.

${ }^{9}$ Gear, C. W., and Leimkuhler, B., "Automatic Integration of Euler-Lagrange Equations with Constraints," Journal of Computational and Applied Mathematics, Vols. 12 and 13, 1985, pp. 77-90.

${ }^{10}$ Chace, M. A., and Smith, D. A., "DAMN-A Digital Computer Program for the Dynamic Analysis of Generalized Mechanical Systems," SAE Paper 710244, Jan. 1971.

${ }^{11}$ Nikravesh, P. E., "Some Methods for Dynamic Analysis of Constrained Mechanical Systems: A Survey," Computer Aided Analysis and Optimization of Mechanical System Dynamics, edited by E. J. Haug, NATO ASI Series, Series F, Vol. 9, Springer-Verlag, Heidelberg, 1984, pp. 351-368.

${ }^{12}$ Baumgarte, J., "Stabilization of Constraints and Integrals of Motion in Dynamical Systems," Computer Methods in Applied Mechanics and Engineering, Vol. 1, 1972, pp. 1-16.

${ }^{13}$ Baumgarte, J., "A New Method of Stabilization for Holonomic Constraints," ASME Journal of Applied Mechanics, Vol. 50, 1983, pp. 869-870.

${ }^{14}$ Chang, C. O., "Dynamic Analysis and Optimum Design of Mechanical Systems with Constraint Violation Stabilization Method," Ph.D. Dissertation, Dept. of Mechanical Engineering, Univ. of Iowa, Aug. 1984.

${ }^{15}$ Park, K. C., and Chiou, J. C., "Stabilization of Computational Procedures for Constrained Dynamical Systems," Journal of Guidance, Control, and Dynamics, Vol. 11, No. 4, 1988, pp. 365-370.

${ }^{16}$ Housner, A., Analog and Analog/Hybrid Computer Programming, Prentice-Hall, Englewood Cliffs, NJ, 1971.

${ }^{17}$ Fogarty, L. E., and Howe, R. M., "Space Trajectory Computations at the University of Michigan,' Simulation, Vol. 6, No. 4, 1966, pp. 220-226.

${ }^{18}$ Fogarty, L. E., and Howe, R. M., "Axis Systems for Analog and Digital Computation of Space and Reentry Trajectories," Application Rept., Applied Dynamics, Ann Arbor, MI, Sept. 1963.

${ }^{19}$ Yoon, S., "'Real-Time Simulation of Constrained Dynamic Systems," Ph.D. Dissertation, Dept. of Aerospace Enginering, Univ. of Michigan, Ann Arbor, MI, 1990. 